\title{
Low expression of Rap1GAP is associated with epithelial- mesenchymal transition (EMT) and poor prognosis in gastric cancer
}

\author{
Ya Yang ${ }^{1,4, *}$, Jia Zhang ${ }^{1, *}$, Yan Yan ${ }^{1}$, Hui Cai ${ }^{2}$, Min Li $^{1}$, Kai Sun ${ }^{1}$, Jizhao Wang ${ }^{1}$, Xu \\ Liu ${ }^{1}$, Jiansheng Wang ${ }^{1}$, Xiaoyi Duan ${ }^{3}$ \\ ${ }^{1}$ The Second Department of Thoracic Surgery, The First Affiliated Hospital of Xi'an Jiaotong University, Xi'an 710061, Shaanxi, \\ China \\ ${ }^{2}$ Department of Vascular Surgery, The First Affiliated Hospital of Xi'an Jiaotong University, Xi'an 710061, Shaanxi, China \\ ${ }^{3}$ Department of Radiology, The First Affiliated Hospital of Xi'an Jiaotong University, Xi'an 710061, Shaanxi, China \\ ${ }^{4}$ Department III of Radiation Oncology, The First Affiliated Hospital of Zhengzhou University, Zhengzhou 450000, Henan, China \\ *These authors have contributed equally to this work \\ Correspondence to: Xiaoyi Duan, email: duanxy@mail.xjtu.edu.cn \\ Jiansheng Wang, email: wangjsh@mail.xjtu.edu.cn \\ Keywords: gastric cancer, RAPI GTPase activating protein (Rapl GAP), E-cadherin, Matrix metalloproteinase-2, prognosis \\ Received: October 19,2015 Accepted: November 21, $2016 \quad$ Published: December 21, 2016
}

\section{ABSTRACT}

Rap1GAP is a crucial tumor suppressor, but its role in gastric cancer (GC) is little investigated. In this study, we found that the expression of Rap1GAP was decreased in GC. Low expression of Rap1GAP was positively correlated with advanced pTNM stage, Borrmann types, tumor diameter and poor prognosis in patients with GC. Low expression of Rap1GAP correlated with loss of E-cadherin expression, and anomalous positivity of MMP2 expression. Multivariate analysis showed that low expression of Rap1GAP was an independent prognostic factor. Ectopic expression of Rap1GAP impaired cell migration and invasion, promoted the expression of E-cadherin and decreased the expression of MMP2. These results suggest that Rap1GAP functions as a novel suppressor of EMT and tumor metastasis in GC, and loss of Rap1GAP predicts poor prognosis in GC.

\section{INTRODUCTION}

Rap1GAP, a 663-amino-acid protein with a molecular weight of $73 \mathrm{kDa}$, is the first identified member of the family of GTPase-activating proteins (GAPs) [1, 2]. Rap1GAP is a critical tumor suppressor gene that is downregulated in multiple cancers such as prostate cancer [3], breast cancer [4], oropharyngeal squamous cell carcinoma [5], pancreatic cancer [6], thyroid carcinoma [7], melanoma [8], colorectal cancer [9], B-cell lymphomas [10], kidney cancer [11], and acute myeloid leukemia [12]. Firstly, overexpression of Rap1GAP was found to inhibit the proliferation of rat thyroid cells [13]. Thereafter, overexpression of Rap1GAP in cancer cells impairs cell migration and invasion in vitro [5, 6, 9, 13-15], and inhibits tumor formation and metastasis in vivo $[6,14,16,17]$. Rap1GAP also impairs cell-matrix adhesion in the absence of effects on cell-cell adhesion [15].
The molecular mechanism of Rap1GAP downregulation in cancers is poorly understood. In human colon carcinoma cells, downregulation of Rap1GAP promotes tumor progression by altering cell/matrix and cell/cell adhesion [9]. In thyroid cells, overexpression of Rap1GAP impairs TSH/cAMP-stimulated p70S6 kinase activity and cell proliferation [13]. Rap1GAP impairs cell-matrix adhesion in the absence of effects on cell-cell adhesion [15]. In breast cancer, genomic mutation of Rap1GAP plays an important role in cancer progression [18]. However, in head and neck squamous cell carcinoma, Rap1GAP promotes invasion via induction of MMP9 secretion [19]. In myeloid leukemia cell lines, Rap1GAP promotes leukemia cell differentiation and apoptosis, but increases leukemia cell invasion in vitro by the upregulation of MMP9 [12]. To date, no study has reported Rap1GAP expression in GC patients. Therefore, in the present study, the expression of Rap1GAP in GC 
and its association with clinicopathological parameters and prognosis were investigated.

Epithelial-mesenchymal transition (EMT) is a complex biological program in which epithelial cells undergo a dramatic morphological change and switch to form mesenchymal cells. This switch occurs along with a reduction of epithelial marker proteins, such as E-cadherin, and an increase of mesenchymal markers, such as N-cadherin [20-22]. Substantial evidence suggests that EMT plays a critical role during embryonic development and cancer progression, the latter being involved in tumor invasion, metastasis, apoptosis and senescence resistance [23-25]. EMT is not only deemed a player in the initiation of the invasion and metastasis and but also a sign of powerful capacity to invade and metastasize [23, $26,27]$. Hence, the expression of E-cadherin and MMP2 were evaluated in GC, and the association of Rap1GAP with E-cadherin and MMP2 was also investigated. Furthermore, we explored the effect of Rap1GAP in vitro, and the corresponding changes in E-cadherin and MMP2 expression, and in migration and invasion capacity of GC cells.

\section{RESULTS}

\section{Expression of Rap1GAP, E-cadherin and MMP2 in $\mathbf{G C}$}

The expression of Rap1GAP was determined in formalin-fixed, paraffin-embedded cancer tissues and para-carcinoma tissues by immunohistochemistry. Figure
$1 \mathrm{~A}$ as a negative control, as predicted, Rap1GAP was highly expressed in the normal follicular epithelial cells and was observed primarily in the cytoplasm (Figure 1B); however, Rap1GAP was not expressed in the stromal cells, endothelial cells and lymphocytes. Although Rap1GAP was detected in the tumor cells, there was a marked decrease in the staining intensity in these cells compared to the para-carcinoma tissues. Most of the GC samples were graded as negative (117 of $178,65.73 \%$ ), and $34.27 \%$ were positive (Table 1). These results are summarized in Figure $1 \mathrm{E}$ and confirmed a significant downregulation of Rap1GAP levels in GC.

To investigate whether the loss of Rap1GAP expression in GC accounts for its EMT-like features and metastasis, we further analyzed the correlations between Rap1GAP and E-cadherin and MMP2. In GC, the epithelial protein loss frequency was $60.67 \%$ (108/178) for E-cadherin (Table 1 and Figure 1C, 1F), and aberrant metastasis protein expression frequencies were $74.15 \%$ (132/178) for MMP2 (Table 1 and Figure 1D), which was detected primarily in the cytoplasm. In addition, the expression levels of Rap1GAP and E-cadherin protein in GC cells were notably increased compared to $293 \mathrm{~T}$ cells. Conversely, MMP2 was decreased in GC cells (Figure 3).

\section{Correlation between Rap1GAP expression with clinicopathological characteristics and E-cadherin and MMP2 expression in GC}

The correlation between the Rap1GAP expression levels and the clinicopathological characteristics in GC
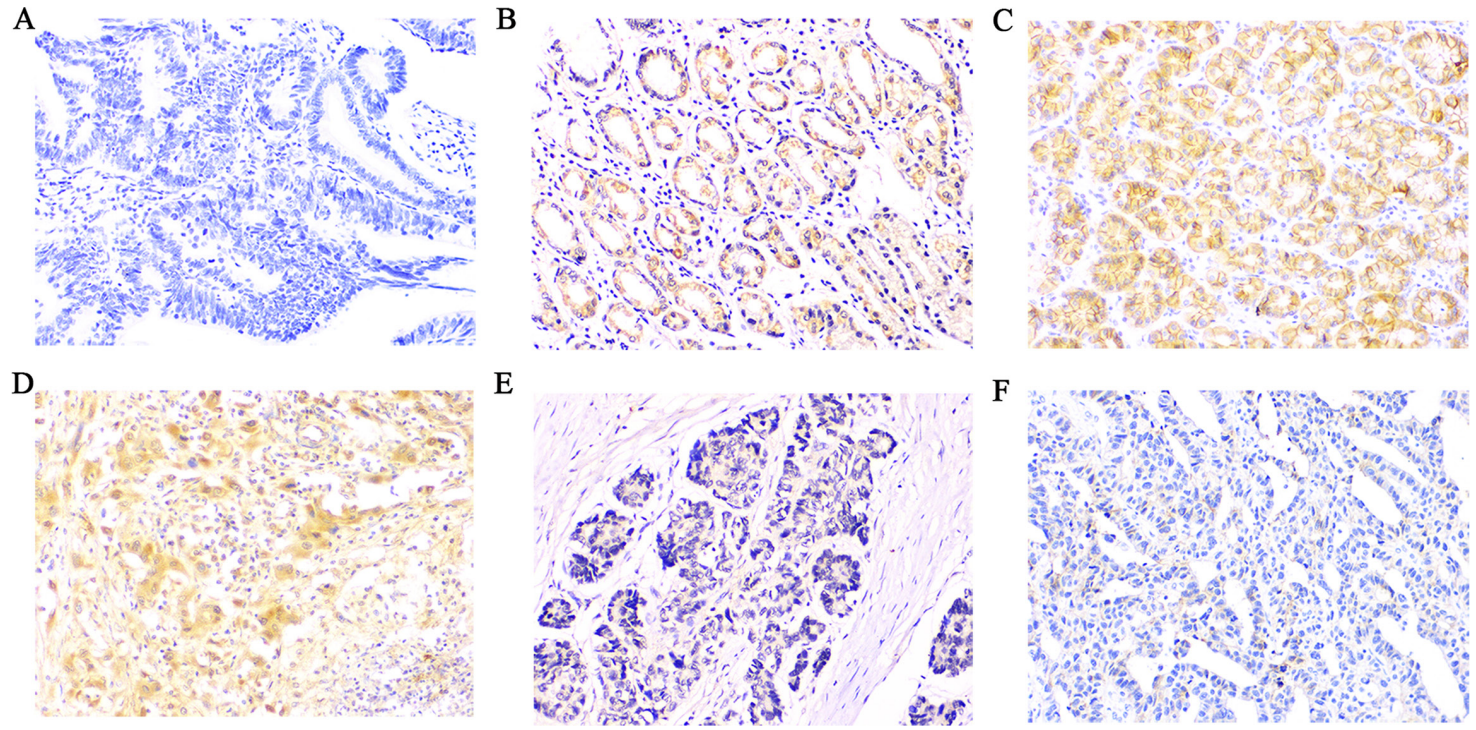

$\mathrm{E}$

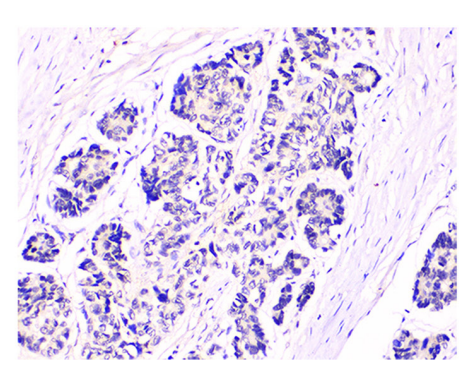

$\mathrm{F}$

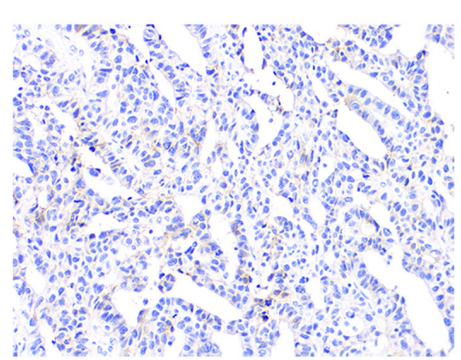

Figure 1: Immunohistochemical expression of Rap1GAP, E-cadherin and MMP2 in GC and para-carcinoma tissues. A. Slides without primary antibody served as the negative control in GC tissues, B, E. Typical immunohistologic features with Rap1GAP expression in para-carcinoma and GC tissues, the Rap1GAP staining localized predominantly in the cytoplasm. C, F. Typical immunohistologic features with E-cadherin expression in para-carcinoma and GC tissues. D. Immunostaining for MMP2 was performed in GC; Magnifications, $\times 200$. 
was further analyzed, the results are summarized in Table 2. Low-expression of Rap1GAP was closely correlated with the pTNM stage, nodal involvement, metastasis, Borrmann types and tumor diameter, but not to the patients' age, gender, the depth of invasion and histology. Moreover, the pTNM stage, depth of invasion, nodal involvement and histology were significantly correlated with a low expression of E-cadherin. High level of MMP2 was significantly correlated with the pTNM stage, depth of invasion, nodal involvement and metastasis (Table 2). Additionally, low expression of Rap1GAP was positively correlated with E-cadherin $(\mathrm{r}=0.433, \mathrm{P}<0.001)$ and negatively associated with MMP2 expression $(r=-0.403$, $\mathrm{P}<0.001$ ) (Table 3).

\section{Survival analysis}

Survival curves were calculated using the KaplanMeier method and compared using the log-rank test. The patients with low Rap1GAP expression showed a more unfavorable prognosis than those with high expression $(\mathrm{P}<0.001)$ (Figure 2A and Table 4). The high levels of MMP2 expression had a statistically significant correlation with poor overall survival $(\mathrm{P}<0.001)$ (Figure $2 \mathrm{C}$ and Table 4); however, the low expression of E-cadherin was associated with no significant difference in the outcomes $(\mathrm{P}=0.091)$ (Figure $2 \mathrm{~B}$ and Table 4$)$. Moreover, as seen in Table 5, multivariate Cox analysis showed that a low expression level of Rap1GAP was an independent prognostic factor for patients with $\mathrm{GC}(\mathrm{P}<0.001)$

\section{Overexpression Rap1GAP promoted the expression of E-cadherin and suppressed the expression of MMP2 in GC cells}

Rap1GAP was associated with expression of EMT features and aggressive phenotype of tumors. To illuminate Rap1GAP function in GC cells, BGC823 and SGC7901 cells are easy to metastasize distally and selected for the following experiments, we then transfected negative

Table 1: Differences in Rap1GAP, E-cadherin and MMP2 between the cancer tissues and para-carcinoma tissues

\begin{tabular}{|c|c|c|c|c|c|c|c|c|c|}
\hline \multirow{2}{*}{ Group } & \multicolumn{2}{|c|}{ Rap1GAP } & \multirow{2}{*}{$\mathbf{P}$} & \multicolumn{2}{|c|}{ E-cadherin } & \multirow{2}{*}{$\mathbf{P}$} & \multicolumn{2}{|c|}{ MMP2 } & \multirow{2}{*}{$\mathbf{P}$} \\
\hline & + & - & & + & - & & + & - & \\
\hline cancer & 61 & 117 & & 70 & 108 & & 132 & 46 & \\
\hline para-carcinoma & 133 & 45 & $<0.001$ & 148 & 30 & $<0.001$ & 65 & 113 & $<0.001$ \\
\hline total & 194 & 162 & & 218 & 138 & & 197 & 159 & \\
\hline
\end{tabular}

A

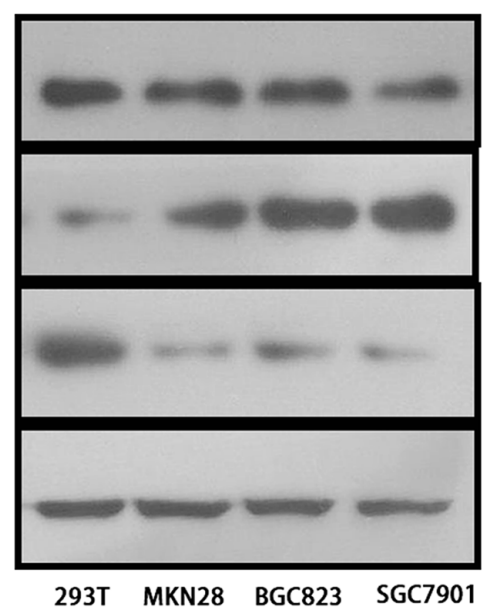

B

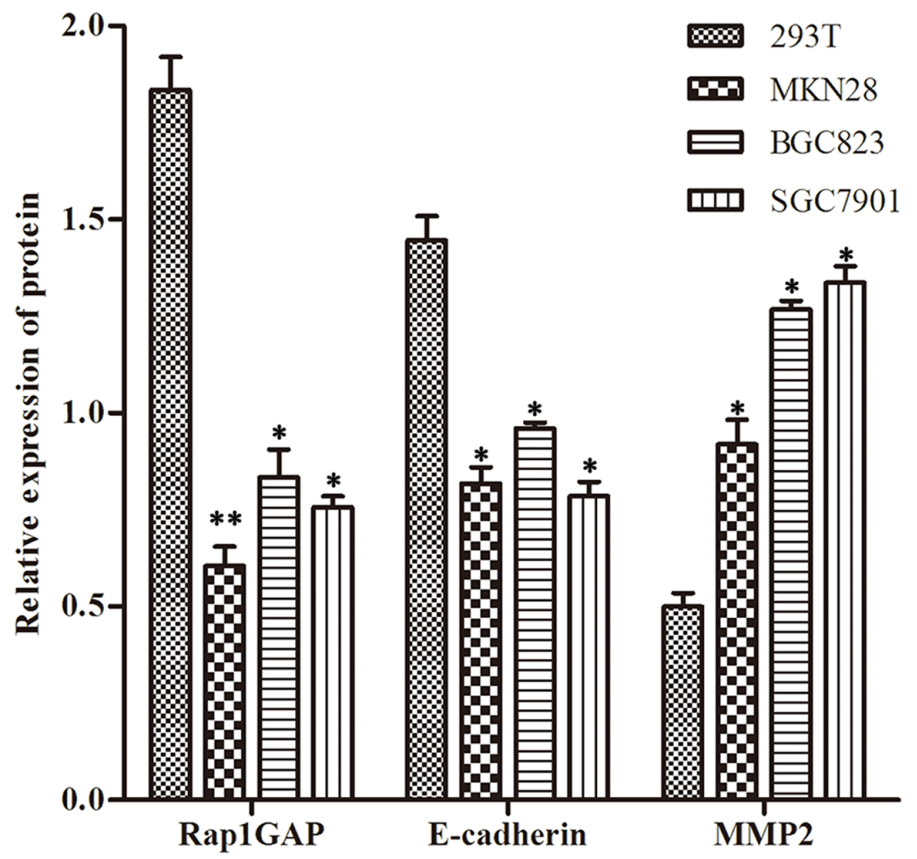

Figure 3: The protein expression of Rap1GAP, E-cadherin and MMP2. Rap1GAP and E-cadherin protein in GC cells were notably lower expression compared to $293 \mathrm{~T}$ cells by western blotting. Conversely, MMP2 was upregulated in GC cells $(* \mathrm{P}<0.05$ and $* * \mathrm{P}$ $<0.01)$ 
Table 2: Correlations between the clinicopathologic variables with Rap1GAP, E-cadherin and MMP2

\begin{tabular}{|c|c|c|c|c|c|c|c|c|c|c|}
\hline \multirow{2}{*}{ Variables } & \multirow{2}{*}{$\mathbf{N}$} & \multicolumn{2}{|c|}{ Rap1GAP } & \multirow{2}{*}{$\mathbf{P}$} & \multicolumn{2}{|c|}{ E-cadherin } & \multirow{2}{*}{$\mathbf{P}$} & \multicolumn{2}{|c|}{ MMP2 } & \multirow{2}{*}{$\mathbf{P}$} \\
\hline & & high & low & & high & low & & high & low & \\
\hline \multicolumn{11}{|l|}{ Gender } \\
\hline male & 125 & 26 & 99 & & 41 & 84 & & 72 & 53 & \\
\hline female & 53 & 16 & 37 & 0.177 & 19 & 34 & 0.694 & 30 & 23 & 0.902 \\
\hline \multicolumn{11}{|l|}{ Age } \\
\hline$\leq 60$ & 94 & 26 & 68 & & 33 & 61 & & 51 & 43 & \\
\hline$>60$ & 84 & 16 & 68 & 0.177 & 27 & 57 & 0.676 & 51 & 33 & 0.384 \\
\hline \multicolumn{11}{|l|}{ TNM } \\
\hline I & 21 & 8 & 13 & & 16 & 5 & & 6 & 15 & \\
\hline II & 34 & 12 & 22 & & 19 & 15 & & 14 & 20 & \\
\hline III & 83 & 6 & 77 & $<0.001$ & 13 & 70 & $<0.001$ & 55 & 28 & 0.002 \\
\hline IV & 40 & 16 & 24 & & 12 & 28 & & 27 & 13 & \\
\hline \multicolumn{11}{|c|}{ Depth of invasion } \\
\hline $\mathrm{T} 1$ & 16 & 6 & 10 & & 11 & 5 & & 3 & 13 & \\
\hline $\mathrm{T} 2$ & 18 & 5 & 13 & & 13 & 5 & & 6 & 12 & \\
\hline $\mathrm{T} 3$ & 67 & 18 & 49 & 0.239 & 20 & 47 & $<0.001$ & 39 & 28 & $<0.001$ \\
\hline $\mathrm{T} 4$ & 77 & 13 & 64 & & 16 & 61 & & 54 & 23 & \\
\hline \multicolumn{11}{|c|}{ Nodal involvement } \\
\hline N0 & 49 & 18 & 31 & & 33 & 16 & & 18 & 31 & \\
\hline N1 & 36 & 4 & 32 & & 8 & 28 & & 24 & 12 & \\
\hline $\mathrm{N} 2$ & 35 & 4 & 31 & 0.011 & 6 & 29 & $<0.001$ & 21 & 14 & 0.007 \\
\hline N3 & 58 & 16 & 42 & & 13 & 45 & & 39 & 19 & \\
\hline \multicolumn{11}{|l|}{ Metastasis } \\
\hline M0 & 138 & 26 & 112 & & 49 & 89 & & 73 & 65 & \\
\hline M1 & 40 & 16 & 24 & 0.006 & 11 & 29 & 0.346 & 29 & 11 & 0.027 \\
\hline \multicolumn{11}{|c|}{ Borrmann type } \\
\hline I & 22 & 10 & 12 & & 9 & 13 & & 11 & 11 & \\
\hline II & 55 & 8 & 47 & & 23 & 32 & & 30 & 25 & \\
\hline III & 62 & 14 & 48 & 0.037 & 20 & 42 & 0.156 & 38 & 24 & 0.778 \\
\hline IV & 39 & 10 & 29 & & 8 & 31 & & 23 & 16 & \\
\hline \multicolumn{11}{|c|}{ Differentiation } \\
\hline high & 12 & 4 & 8 & & 8 & 4 & & 6 & 6 & \\
\hline moderate & 57 & 15 & 42 & 0.538 & 26 & 31 & 0.001 & 28 & 29 & 0.226 \\
\hline poor & 109 & 23 & 86 & & 26 & 83 & & 68 & 41 & \\
\hline \multicolumn{11}{|c|}{ Tumor diameter } \\
\hline$<3 \mathrm{~cm}$ & 44 & 16 & 28 & & 21 & 23 & & 20 & 24 & \\
\hline $3 \mathrm{~cm}-5 \mathrm{~cm}$ & 54 & 7 & 47 & 0.025 & 18 & 36 & 0.053 & 33 & 21 & 0.187 \\
\hline$>5 \mathrm{~cm}$ & 80 & 19 & 61 & & 21 & 59 & & 49 & 31 & \\
\hline
\end{tabular}


control (NC) and Rap1GAP CRISPR Activation Plasmid into GC cells. The expression of both E-cadherin mRNA and protein was increased compared to $\mathrm{NC}(\mathrm{P}<0.05$; Figure 4); on the contrary, MMP2 was inhibited at the mRNA and protein level ( $<<0.05$; Figure 4$)$. Rap1GAP promoted the expression of E-cadherin and suppressed the expression of MMP2 in GC cells. This suggested that E-cadherin and MMP2 might be the downstream genes of Rap1GAP.

\section{Overexpression Rap1GAP repressed the migration and invasion capacity of tumor cells}

Overexpression Rap1GAP cells were cultivated for 24 hours. Then, the migration assay and transwell invasion assay were used to detect the capacity of migration and invasion of Rap1GAP cells. As shown in Figure 5, both migration and invasive cell number were decreased following overexpression of Rap1GAP by Rap1GAP CRISPR Activation Plasmid ( $\mathrm{P}<0.05)$. Hence, Rap1GAP played an important role in the migration and invasion capacity of tumor cells.

\section{DISCUSSION}

Rap1GAP is a Rap1 GTPase-activating protein that inhibits Rap1 activity by converting the active, GTP-bound from to the inactive, GDP-bound form [28]. Increasing evidences suggest that Rap1GAP function as a tumor suppressor through inhibiting proliferation, impairing cell invasion and metastasis and accelerating apoptosis [8, 14, 15, 17]; however, some studies indicated that an increased expression of Rap1GAP promoted tumor invasion and induction via MMP9 secretion [6, 12]. A critical question raised was whether Rap1GAP expression is clinically correlated with GC progression. In this study, we found that Rap1GAP expression in cancer tissues was lower than adjacent non-tumor tissues. Lower or lost expression of Rap1GAP has been reported in pancreatic cancer [6], thyroid tumors [7] and melanoma [8]. The low expression level of Rap1GAP was closely correlated with the pTNM stage, nodal involvement, metastasis, Borrmann types, tumor diameter and poor overall survival. Our findings of Rap1GAP low expression in both the GC

Table 3: Association between Rap1GAP with E-cadherin and MMP2

\begin{tabular}{lcccc}
\hline & \multicolumn{2}{c}{ Rap1GAP } & r & P \\
\cline { 2 - 3 } & high & low & & \\
\hline E-cadherin & 30 & 30 & 0.433 & $<0.001$ \\
high & 12 & 106 & & \\
low & & & & $<0.001$ \\
MMP2 & 9 & 93 & -0.403 & \\
high & 33 & 43 & & \\
low & & & & \\
\hline
\end{tabular}
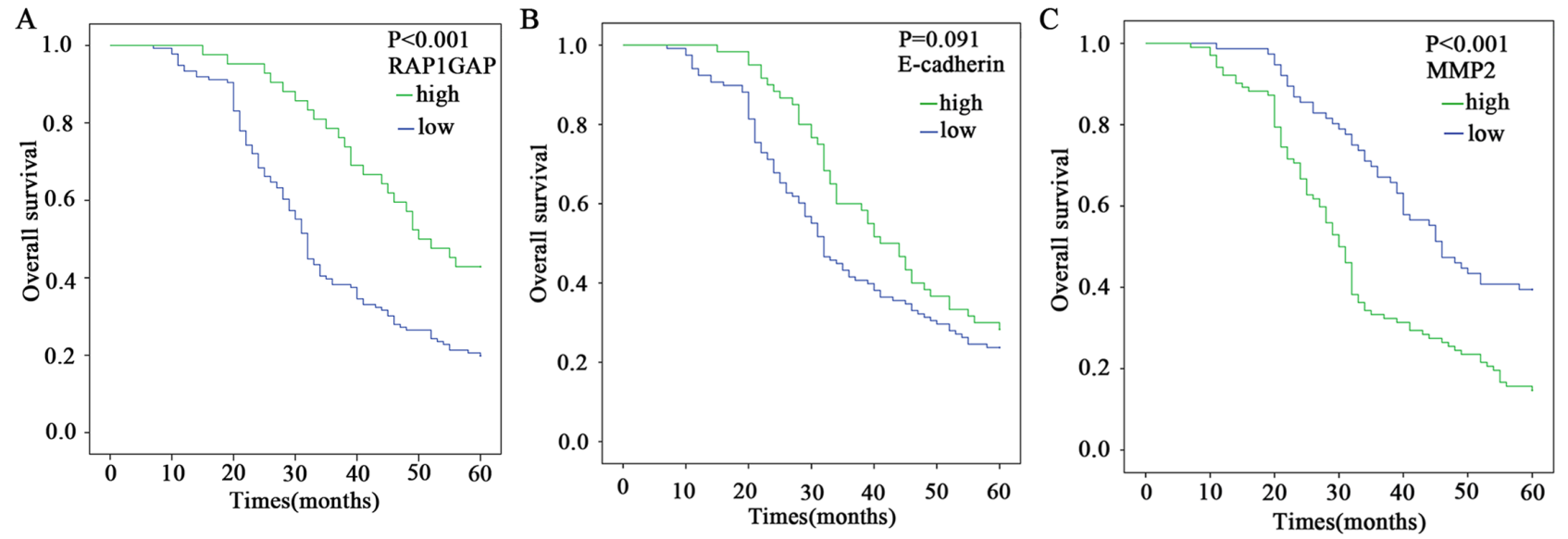

Figure 2: Kaplan-Meier survival analysis of Rap1GAP, E-cadherin and MMP2 expression levels. The cumulative overall survival differences between the patients with high and low levels of protein expression. The P value was obtained using the log-rank test of the difference. A. Rap1GAP; B. E-cadherin; C. MMP2. 


\begin{tabular}{|c|c|c|c|c|}
\hline \multirow{2}{*}{ Variables } & \multirow{2}{*}{$\mathbf{N}$} & \multicolumn{2}{|c|}{ Overall survival } & \multirow{2}{*}{$\mathbf{P}$} \\
\hline & & Median \pm SE & $95 \% \mathrm{CI}$ & \\
\hline \multicolumn{5}{|l|}{ Rap1GAP } \\
\hline high & 42 & $50.00 \pm 4.32$ & $41.53-58.46$ & \\
\hline low & 136 & $32.00 \pm 0.89$ & $30.37-33.62$ & $<0.001$ \\
\hline \multicolumn{5}{|l|}{ E-cadherin } \\
\hline high & 60 & $41.00 \pm 3.32$ & $34.49-47.50$ & \\
\hline low & 118 & $32.00 \pm 1.80$ & $28.45-35.54$ & 0.091 \\
\hline \multicolumn{5}{|l|}{ MMP2 } \\
\hline high & 102 & $30.00 \pm 1.12$ & $27.80-32.19$ & \\
\hline low & 76 & $46.00 \pm 2.72$ & $40.66-51.33$ & $<0.001$ \\
\hline \multicolumn{5}{|l|}{ Gender } \\
\hline male & 125 & $34.00 \pm 2.97$ & $28.16-39.83$ & \\
\hline female & 53 & $38.00 \pm 3.63$ & $30.86-45.13$ & 0.973 \\
\hline \multicolumn{5}{|l|}{ Age } \\
\hline$\leq 60$ & 94 & $40.00 \pm 3.87$ & $32.40-47.59$ & \\
\hline$>60$ & 84 & $32.00 \pm 1.13$ & $29.77-34.22$ & 0.223 \\
\hline \multicolumn{5}{|l|}{ TNM } \\
\hline I & 21 & $49.00 \pm 3.21$ & 42.69-66.71 & \\
\hline II & 34 & $45.00 \pm 5.83$ & $33.57-56.42$ & \\
\hline III & 83 & $34.00 \pm 2.27$ & $29.53-38.46$ & \\
\hline IV & 40 & $29.00 \pm 1.57$ & 25.91-32.08 & $<0.001$ \\
\hline \multicolumn{5}{|c|}{ Depth of invasion } \\
\hline $\mathrm{T} 1$ & 16 & $46.00 \pm 8.66$ & $29.01-62.98$ & \\
\hline $\mathrm{T} 2$ & 18 & $52.00 \pm 15.91$ & $20.81-83.18$ & \\
\hline T3 & 67 & $35.00 \pm 4.60$ & $25.98-44.01$ & \\
\hline $\mathrm{T} 4$ & 77 & $31.00 \pm 1.45$ & $28.14-33.86$ & 0.005 \\
\hline \multicolumn{5}{|c|}{ Nodal involvement } \\
\hline No & 49 & $55.00 \pm 9.79$ & $35.79-74.20$ & \\
\hline N1 & 36 & $43.00 \pm 7.80$ & $27.22-58.78$ & \\
\hline $\mathrm{N} 2$ & 35 & $36.00 \pm 3.54$ & $29.04-42.95$ & \\
\hline $\mathrm{N} 3$ & 58 & $29.00 \pm 2.53$ & 24.03-33.96 & $<0.001$ \\
\hline \multicolumn{5}{|l|}{ Metastasis } \\
\hline M0 & 138 & $40.00 \pm 3.91$ & $32.32-47.67$ & \\
\hline M1 & 40 & $29.00 \pm 1.57$ & $25.91-32.08$ & $<0.001$ \\
\hline \multicolumn{5}{|l|}{ Borrmann type } \\
\hline I & 22 & $39.00 \pm 7.62$ & $24.06-53.93$ & \\
\hline II & 55 & $40.00 \pm 3.69$ & $32.76-47.23$ & \\
\hline III & 62 & $34.00 \pm 3.50$ & $27.14-40.85$ & \\
\hline IV & 39 & $30.00 \pm 1.77$ & $27.51-34.48$ & 0.502 \\
\hline \multicolumn{5}{|l|}{ Differentiation } \\
\hline high & 12 & $40.00 \pm 6.06$ & $28.11-51.88$ & \\
\hline moderate & 57 & $39.00 \pm 6.11$ & $26.97-51.02$ & \\
\hline poor & 109 & $32.00 \pm 1.74$ & $28.59-35.41$ & 0.107 \\
\hline \multicolumn{5}{|c|}{ Tumor diameter } \\
\hline$<3 \mathrm{~cm}$ & 44 & $46.00 \pm 5.20$ & $35.79-56.20$ & \\
\hline $3 \mathrm{~cm}-5 \mathrm{~cm}$ & 54 & $34.00 \pm 1.83$ & $30.40-37.51$ & \\
\hline$>5 \mathrm{~cm}$ & 80 & $32.00 \pm 0.98$ & $30.06-33.93$ & 0.019 \\
\hline
\end{tabular}


Table 5: Multivariate Cox Proportional Hazards Analysis for Overall Survival

\begin{tabular}{lccc}
\hline \multirow{2}{*}{ Variables } & \multicolumn{2}{c}{ Overall survival } & P \\
\cline { 2 - 4 } Nodal involvement & $\mathbf{R R}$ & $\mathbf{9 5 \% \mathbf { C I }}$ & 0.016 \\
Rap1GAP & 1.249 & $1.042-1.498$ & $<0.001$ \\
MMP2 & 2.578 & $1.531-4.341$ & 0.034 \\
Age & 1.502 & $1.032-2.188$ & 0.611 \\
Gender & 0.903 & $0.609-1.339$ & 0.223 \\
Depth of invasion & 0.836 & $0.626-1.115$ & 0.293 \\
Metastasis & 1.073 & $0.720-1.599$ & 0.057 \\
TNM & 1.151 & $0.894-1.476$ & 0.179 \\
Borrmann type & 1.064 & $0.698-1.578$ & 0.259 \\
Differentiation & 0.844 & $0.637-1.129$ & 0.622 \\
Tumor diameter & 0.930 & $0.674-1.285$ & 0.717 \\
\hline
\end{tabular}

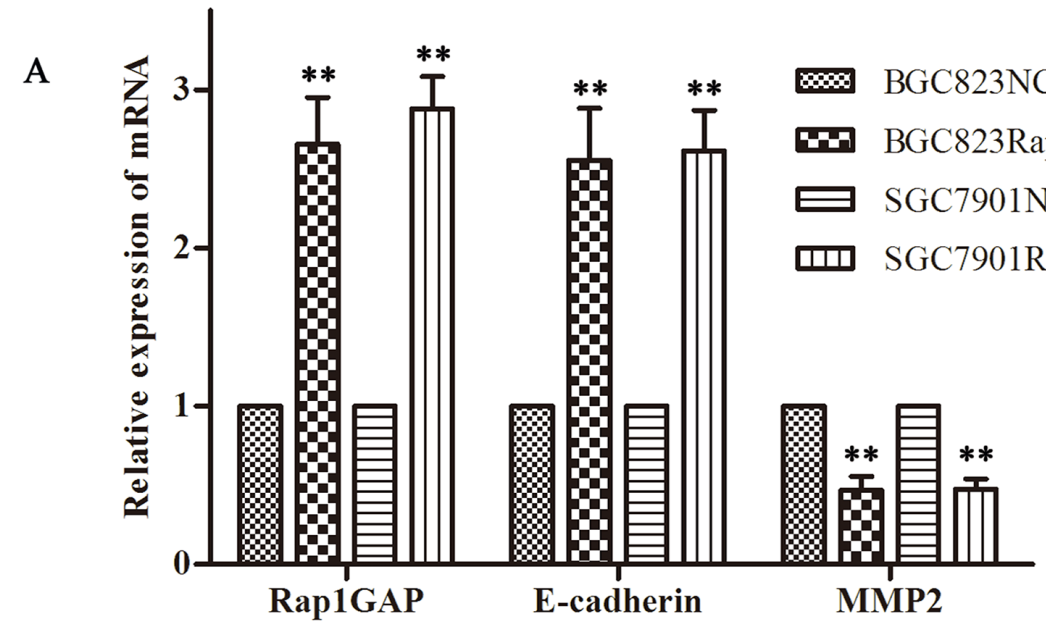

B
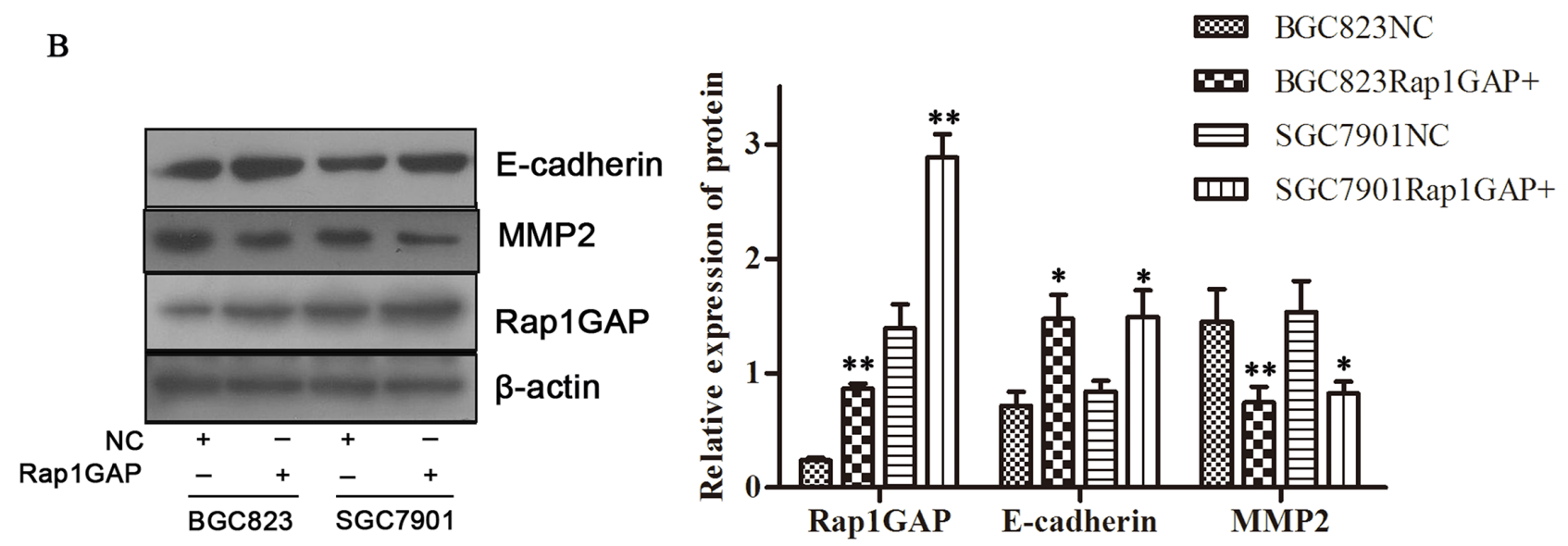

Figure 4: Overexpression Rap1GAP promoted the expression of E-cadherin and suppressed the expression of MMP2 in GC cells. The GC cells were grown and transfected with the negative control (NC) or Rap1GAP CRISPR Activation Plasmid. Overexpression Rap1GAP improved the expression of E-cadherin (A, B) and, inversely, suppressed the expression of MMP2 (A, B) compared to NC group in mRNA level and protein level. ( $* \mathrm{P}<0.05$ and $* * \mathrm{P}<0.01)$. 
samples and the cell lines indicate a role for Rap1GAP in gastric tumorigenesis.

The downregulation of Rap1GAP was influenced by various mechanisms, and RaplGAP may exert cell type- and context-specific functions. In thyroid carcinoma cells, Rap1GAP expression was abolished by Ras transformation, promoter hypermethylation and loss of heterozygosity, and the downregulation of Rap1GAP promoted cell proliferation, migration and invasion [7, $14,29]$. In the head and neck squamous cell carcinoma (SCC), EZH2 repressed Rap1GAP by facilitating the trimethylationof $\mathrm{H} 3 \mathrm{~K} 27$ and hypermethylation of the Rap1GAP promoter, and the downregulation of Rap1GAP activated the activity of Rap1, and eventually promoted proliferation and invasion [30]. Rap1GAP has been shown to be frequently suppressed in malignant melanoma via promoter hypermethylation, which promoted melanoma cell proliferation, survival and migration [8]. The low expression levels of Rap1GAP in renal cell carcinoma cells was partly due to promoter hypermethylation, and attenuated the invasion of kidney cancer cells [31]. The downregulation of Rap1GAP was affected by various epigenetic mechanisms.

EMT along with a reduction of E-cadherin underlies the initiation of the invasion and metastasis of many human malignancies including GC [24, 32, 33]. One report showed that the overexpression of Rap1GAP decreases the expression of E-cadherin [31], however, our results showed that overexpression of Rap1GAP increased the expression of E-cadherin. Of particular relevance was a report that the E-cadherin protein levels were largely unchanged, but the accumulation of E-cadherin at cell/cell
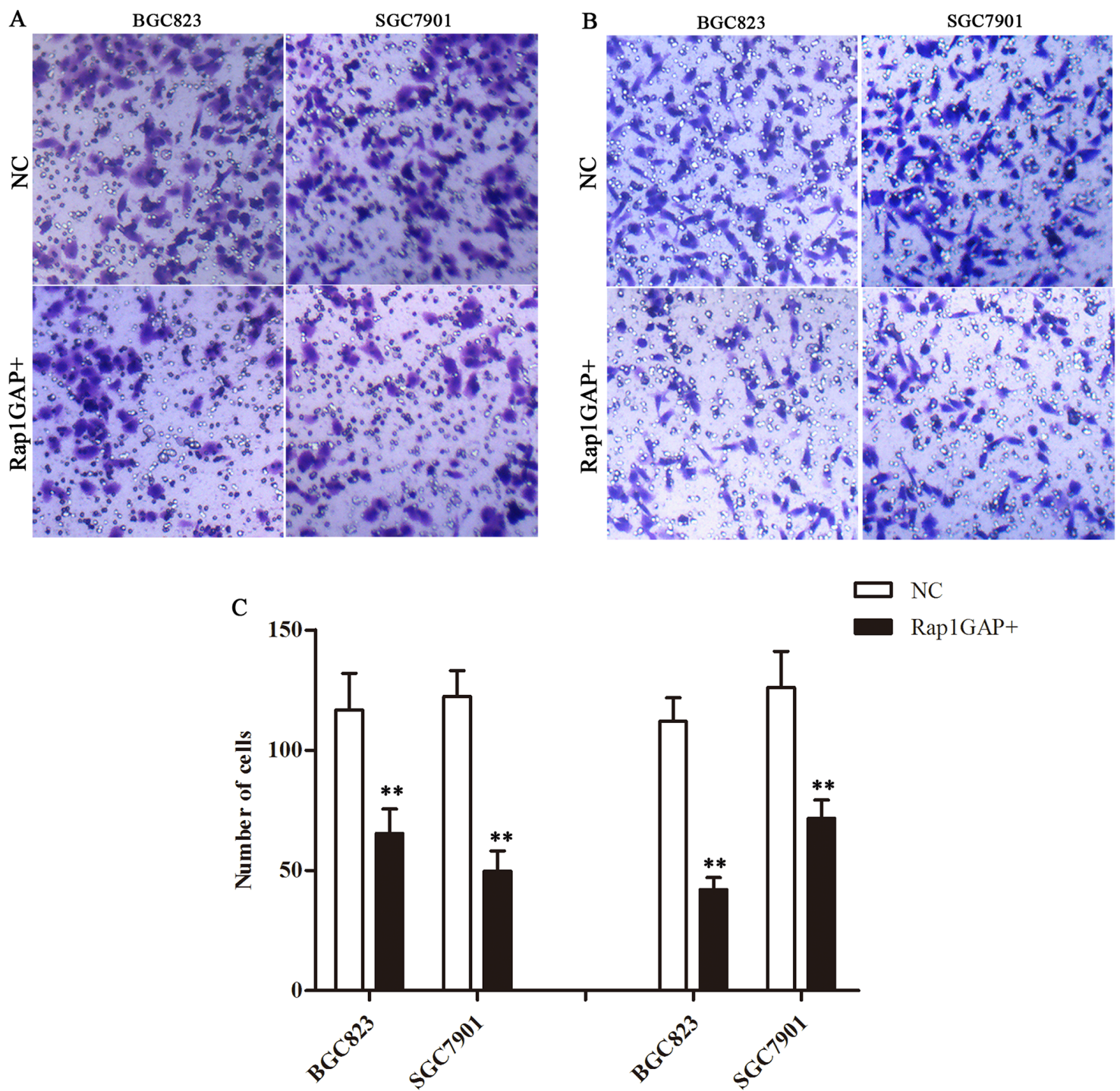

Figure 5: Overexpression Rap1GAP on the regulation of the migration and invasion capacity of tumor cells. Overexpression Rap1GAP repressed the migration A. and invasion B. capacity of GC cells $\mathbf{C}$. The graph shows the quantified data of the assay, migration (left) and invasion (right) $(* * \mathrm{P}<0.01)$. 
junctions reduced in the Rap1GAP-depleted cell lines. The alterations in cell/cell and cell/matrix adhesion are early steps in tumor metastasis supports a role for Rap1GAP depletion in tumor progression [9]. Rap1GAP expression was selectively suppressed in human thyroid carcinoma cell lines that neither unfolded an epithelial morphology nor expressed E-cadherin. The downregulation of Rap1GAP was correlated with the induction of a mesenchymal morphology and migratory behavior [1]. In another study, the overexpression of Rap1GAP did not enhance the dissociation of cell aggregates nor did it impair the accumulation E-cadherin at cell-cell contacts [15]. These data indicate that Rap1GAP is compatible with EMT and that the molecular mechanisms may be more complex than currently appreciated.

Some meta-analyses indicated that MMP2 expression may serve as an independent prognostic factor for GC [34-36]. Our results are similar to these former reports. Little is known about Rap1GAP and MMP2 in $\mathrm{GC}$, and here we addressed a significant correlation between Rap1GAP and MMP2, simultaneously, our results showed that overexpression of Rap1GAP suppressed the expression of MMP2 and the migration and invasion capacity of tumor cells. Only have few studies have investigated the role of Rap1GAP in promoting the invasion of tumor cells via the upregulation of MMP9 secretion $[7,19]$. Further investigations on the regulatory mechanisms of Rap1GAP and MMPs are needed.

In the present study, we found significant correlations between low Rap1GAP expression and the pTNM stage, nodal involvement, metastasis, Borrmann types, tumor diameter and poor overall survival. Rap1GAP is likely to serve as an important tumor suppressor in GC, and its loss during carcinogenesis contributes to tumor progression and metastasis. Rap1GAP was associated with expression of EMT features, aggressive phenotype of tumors and poor prognosis, suggesting Rap1GAP was a potential prognostic marker. Overexpression Rap1GAP promoted the expression of E-cadherin, suppressed the expression of MMP2 and repressed the migration and invasion capacity in GC cells. This suggested that Rap1GAP was one of therapeutic targets for GCs. But the regulation Rap1GAP with E-cadherin and MMP2 was intricate. Thus, further investigations are required.

\section{MATERIALS AND METHODS}

\section{Ethics statement and patients}

We collected tissues specimens of 178 gastric cancer patients from surgical cases in Department of Surgical Oncology, The First Affiliated Hospital, Xi'an Jiaotong University Medical School and Department of Surgical Oncology, the 215th Hospital of Shaanxi province between 2004 and 2009. The patients included 125 male and 53 female patients (ranging from 25 to 81 years of age). All of the patients were assessed according to the system for staging primary tumor/regional lymph nodes/distant metastasis (TNM) described in the AJCC Cancer Staging Manual. None of these 178 patients received neoadjuvant or adjuvant chemotherapy before the operation. The study was approved by the ethics committee and the human research review committee of Xi' an Jiaotong University.

\section{Immunohistochemical staining}

The tissues specimens were fixed in neutral buffered formalin and embedded in paraffin wax. The sections of 4-mm thickness were cut and mounted on charged glass slides. Antigen retrieval was performed using citrate buffer at $\mathrm{pH}$ 6.0. The rabbit polyclonal antibody against Rap1GAP (Biosynthesis Bio-technology), MMP2 (Biosynthesis Bio-technology) and E-cadherin (CST) was performed. The streptavidin-peroxidase technique (Golden Bridge Int) was used. An irrelevant rabbit antiserum served as a negative control. The sections were stained with $0.02 \%$ diaminobenzidine (DAB) solution followed by counterstaining with hematoxylin.

\section{Evaluation of immunohistochemical analysis}

The evaluation of Rap1GAP, E-cadherin and MMP2 expression was performed independently by two experienced pathologists who were blinded to the clinical data with consensus. The staining results were scored semi-quantitatively by calculating the immunostaining intensity and the percentage of positive malignant cells. The percentage of positive malignant cells was determined in at least 5 areas under $400 \times$ magnifications and averaged. The mean percentage was scored as follows: $0(0-5 \%) ; 1(6-25 \%) ; 2(26-50 \%) ; 3(51-75 \%)$, and $4(76-100 \%)$. The staining intensities were scored as follows: no coloring, 0 point; slightly yellow, 1 point; brownish-yellow, 2 points; and tan, 3 points. Finally, the staining score was obtained by calculating the product of the staining intensity and the positive cell percentage, where $\leq 5$ was defined as low expression and $\geq 6$ as high expression.

\section{Cell lines and cell culture}

The 293 T cell and human GC cell lines (MKN28, BGC823 and SGC7901) were obtained from the Cell Bank of Shanghai (Shanghai, China) and cultured in Dulbecco's Modified Eagle Medium (DMEM, Life Technologies)/ high glucose medium supplemented with $10 \%$ heatinactivated newborn calf serum at $37^{\circ} \mathrm{C}$ in a humidified incubator under a $5 \%$ carbon dioxide atmosphere. All of the experiments were performed with cells in the logarithmic phase of growth. 


\section{Western blot analysis}

Whole-cell lysates were prepared from the cell lines with a RIPA lysis buffer kit (Santa Cruz), and the protein concentrations were quantified using a BioRad protein assay (Hercules). An equal amount of protein was separated using $10 \%$ and $12 \%$ SDS-PAGE and transferred to a polyvinylidene fluoride membrane (Millipore). The membranes were then probed with antibodies against the proteinsRap1GAP, E-cadherin and MMP2. The membranes were blocked in tris-buffered saline with tween containing 5\% non-fat dry milk and then incubated overnight with the primary antibody, followed by incubation with the horseradish-peroxidaseconjugated antibodies at room temperature. The blots were developed using a peroxidase reaction and visualized with an ECL detection systems (Millipore). $\beta$-Actin was used as an internal positive control. The blots were analyzed densitometrically using the program Quantity One software (Hercules).

\section{Transfection}

The GC cells were grown and transfected with the NC or Rap1GAP CRISPR Activation Plasmid (Santa Cruz) using Lipofectamine 2000, according to the manufacturer's protocol. After transfection for $48 \mathrm{~h}$, the cells were harvested for RNA isolation and quantitative real-time PCR and western blot analysis of Rap1GAP expression.

\section{RNA isolation and quantitative real-time PCR}

Total cellular RNA was isolated using a TRIzol reagent and reversely transcribed into cDNA using the Superscript II Reverse Transcriptase kit, according to the manufacturers' instructions. The synthesized cDNA was then subjected to qPCR amplification using primers (Rap1GAP, 5'-GCA CTT TCT CGG CAA GGA GCA TTT-3' and 5'-TGA CAT CAT GGT ATG TCC GGC ACT-3'; E-cadherin, 5'-AAG GAG GCG GAG AAG AGG AC-3' and 5'-CGT CG TTA CGA GTC ACT TCA GG-3'; MMP2, 5'-CGC AGT GAC GGA AAG ATG TGG-3' and 5'-AGA GCT CCT GAA TGC CCT TGA-3'; Glyceraldehydes-3-phosphate dehydrogenase (GAPDH), 5'-AGA AGG CTG GGG CTC ATT TG-3' and 5'-AGG GGC CAT CCA CAG TCT TC-3') under the following conditions: $95^{\circ} \mathrm{C}$ for $30 \mathrm{sec}$ and 30 cycles of $95^{\circ} \mathrm{C}$ for 5 $\mathrm{s}, 60^{\circ} \mathrm{C}$ for $30 \mathrm{~s}$, and $72^{\circ} \mathrm{C}$ for $60 \mathrm{sec}$ for 30 cycles. Realtime PCR was performed in a multiplex PCR reaction with GAPDH as an internal control. Real-time PCR was performed with the Bio-Rad Thermocycler (iQ5) using SYBR green reagents (Hercules). The final products were verified by dissociation curves and data were analyzed by the relative quantification method normalized to GAPDH.

\section{Cell migration and invasion}

Cell migration assay was performed using the Boyden chamber containing a membrane with an $8 \mu \mathrm{m}$ pore size (BD Bioscience). Cell invasion studies were also done using the Boyden chamber but membranes were precoated with Matrigel (1 mg/ml; BD Bioscience) matrices. In both cases, cells were seeded in wells at a density of 3-5 $\times 10^{4}$ cells $/ 200 \mu$ in DMEM medium containing $0.2 \%(\mathrm{v} / \mathrm{v})$ FBS in the upper chamber. In the lower chamber, $600 \mu \mathrm{l}$ of DMEM media containing 10\% FBS were added. After $24 \mathrm{~h}$ of incubation at $37^{\circ} \mathrm{C}$ in a $5 \% \mathrm{CO}_{2}$ incubator, the chamber was removed, fixed, and stained with $1 \%$ crystal purple. Cells in the upper chamber were removed using a cotton swab. Cells migrating through the membrane and cells invading the matrix were randomly counted in five visual fields of each membrane at $200 \times$ magnification under a light microscope.

\section{Statistical analysis}

Statistical analysis was performed using the SPSS software package (Version 16.0). A chi-square test was used to test the association of Rap1GAP, E-cadherin and MMP2 expression and the clinicopathological variables. The Spearman's rank correlation coefficient was used for analyzing the association of the E-cadherin and MMP2 expression levels with the Rap1GAP expression levels. Overall survival was defined as the time from the date of surgery to the date of the last follow-up or death from any cause. Survival curves were calculated using the Kaplan-Meier method and compared using the log-rank test. For multivariate analysis, the prognostic factors were analyzed using Cox's proportional hazard model. Student $t$ test or one-way ANOVA was used to compare the normally distributed variables. The results were considered statistically significant if $\mathrm{P}<0.05(\mathrm{P}<$ $\left.0.05^{*}, \mathrm{P}<0.01 * *\right)$.

\section{Abbreviations \\ Rap1GAP, RAP1 GTPase activating protein; GC, gastric cancer; MMP2, Matrix metalloproteinase-2; EMT, Epithelial-mesenchymal transition; TNM, primary tumor/regional lymph nodes/distant metastasis; DAB, diaminobenzidine; DMEM, Dulbecco's Modified Eagle Medium; NC, negative control}

\section{ACKNOWLEDGMENTS}

This study was supported in part by the National Natural Science Foundation of China (no.81471710), and by the science technology research and development projects of Shaanxi (no.2014KW23-02). 


\section{CONFLICTS OF INTEREST}

The authors declare that they have no conflicts of interest.

\section{REFERENCES}

1. Rubinfeld B, Munemitsu S, Clark R, Conroy L, Watt K, Crosier WJ, McCormick F, Polakis P. Molecular cloning of a GTPase activating protein specific for the Krev-1 protein p21rap1. Cell. 1991; 65:1033-42.

2. Frische EW, Zwartkruis FJ. Rap1, a mercenary among the Ras-like GTPases. Dev Biol. 2010; 340:1-9.

3. Bailey CL, Kelly P, Casey PJ. Activation of Rap1 promotes prostate cancer metastasis. Cancer Res. 2009; 69:4962-8.

4. Kato H, Liao Z, Mitsios JV, Wang HY, Deryugina EI, Varner JA, Quigley JP, Shattil SJ. The primacy of beta1 integrin activation in the metastatic cascade. PLoS One. 2012; 7:e46576.

5. Zhang Z, Mitra RS, Henson BS, Datta NS, McCauley LK, Kumar P, Lee JS, Carey TE, D'Silva NJ. Rap1GAP inhibits tumor growth in oropharyngeal squamous cell carcinoma. Am J Pathol. 2006; 168:585-96.

6. Zhang L, Chenwei L, Mahmood R, van Golen K, Greenson J, Li G, D'Silva NJ, Li X, Burant CF, Logsdon CD, Simeone DM. Identification of a putative tumor suppressor gene Rap1GAP in pancreatic cancer. Cancer Res. 2006; 66:898-906.

7. Tsygankova OM, Prendergast GV, Puttaswamy K, Wang Y, Feldman MD, Wang H, Brose MS, Meinkoth JL. Downregulation of Rap1GAP contributes to Ras transformation. Mol Cell Biol. 2007; 27:6647-58.

8. Zheng H, Gao L, Feng Y, Yuan L, Zhao H, Cornelius LA. Down-regulation of Rap1GAP via promoter hypermethylation promotes melanoma cell proliferation, survival, and migration. Cancer Res. 2009; 69:449-57.

9. Tsygankova OM, Ma C, Tang W, Korch C, Feldman MD, Lv Y, Brose MS, Meinkoth JL. Downregulation of Rap1GAP in human tumor cells alters cell/matrix and cell/ cell adhesion. Mol Cell Biol. 2010; 30:3262-74.

10. Wang LD, Zhou FY, Li XM, Sun LD, Song X, Jin Y, Li JM, Kong GQ, Qi H, Cui JA, Zhang LQ, Yang JZ, Li $\mathrm{JL}$, et al. Genome-wide association study of esophageal squamous cell carcinoma in Chinese subjects identifies susceptibility loci at PLCE1 and C20 or f54. Nat Genet. 2010; 42:759-U46.

11. Wu J, Zhang Y, Frilot N, Kim JI, Kim WJ, Daaka Y. Prostaglandin E2 regulates renal cell carcinoma invasion through the EP4 receptor-Rap GTPase signal transduction pathway. J Biol Chem. 2011; 286:33954-62.

12. Qiu T, Qi X, Cen J, Chen Z. Rap1GAP alters leukemia cell differentiation, apoptosis and invasion in vitro. Oncol Rep. 2012; 28:622-8.
13. Tsygankova OM, Feshchenko E, Klein PS, Meinkoth JL. Thyroid-stimulating hormone/cAMP and glycogen synthase kinase 3 beta elicit opposing effects on Rap1GAP stability. J Biol Chem. 2004; 279:5501-7.

14. Zuo H, Gandhi M, Edreira MM, Hochbaum D, Nimgaonkar VL, Zhang P, Dipaola J, Evdokimova V, Altschuler DL, Nikiforov YE. Downregulation of Rap1GAP through epigenetic silencing and loss of heterozygosity promotes invasion and progression of thyroid tumors. Cancer Res. 2010; 70:1389-97.

15. Vuchak LA, Tsygankova OM, Meinkoth JL. Rap1GAP impairs cell-matrix adhesion in the absence of effects on cell-cell adhesion. Cell Adh Migr. 2011; 5:323-31.

16. Bos JL, de Rooij J, Reedquist KA. Rap1 signalling: adhering to new models. Nat Rev Mol Cell Biol. 2001; 2:369-77.

17. Tsygankova OM, Wang H, Meinkoth JL. Tumor cell migration and invasion are enhanced by depletion of Rap1 GTPase-activating protein (Rap1GAP). J Biol Chem. 2013; 288:24636-46

18. Sjoblom T, Jones S, Wood LD, Parsons DW, Lin J, Barber TD, Mandelker D, Leary RJ, Ptak J, Silliman N, Szabo $\mathrm{S}$, Buckhaults P, Farrell C, et al. The consensus coding sequences of human breast and colorectal cancers. Science. 2006; 314:268-74.

19. Mitra RS, Goto M, Lee JS, Maldonado D, Taylor JMG, Pan QT, Carey TE, Bradford CR, Prince ME, Cordell KG, Kirkwood KL, D'Silva NJ. Rap1GAP promotes invasion via induction of matrix metalloproteinase 9 secretion, which is associated with poor survival in low N-stage squamous cell carcinoma. Cancer Res. 2008; 68:3959-69.

20. Tsai JH, Yang J. Epithelial-mesenchymal plasticity in carcinoma metastasis. Genes Dev. 2013; 27:2192-206.

21. Gao D, Vahdat LT, Wong S, Chang JC, Mittal V. Microenvironmental regulation of epithelial-mesenchymal transitions in cancer. Cancer Res. 2012; 72:4883-9.

22. Lamouille S, Xu J, Derynck R. Molecular mechanisms of epithelial-mesenchymal transition. Nat Rev Mol Cell Biol. 2014; 15:178-96.

23. Thiery JP, Acloque H, Huang RY, Nieto MA. Epithelialmesenchymal transitions in development and disease. Cell. 2009; 139:871-90.

24. Watanabe T, Takahashi A, Suzuki K, Kurusu-Kanno M, Yamaguchi K, Fujiki H, Suganuma M. Epithelialmesenchymal transition in human gastric cancer cell lines induced by TNF-alpha-inducing protein of Helicobacter pylori. Int J Cancer. 2014; 134:2373-82.

25. Savagner P. The epithelial-mesenchymal transition (EMT) phenomenon. Ann Oncol. 2010; 21:vii89-92.

26. Hainaut P, Plymoth A. Targeting the hallmarks of cancer: towards a rational approach to next-generation cancer therapy. Curr Opin Oncol. 2013; 25:50-1. 
27. Hanahan D, Weinberg RA. Hallmarks of cancer: the next generation. Cell. 2011; 144:646-74.

28. Bos JL, Rehmann H, Wittinghofer A. GEFs and GAPs: critical elements in the control of small G proteins. Cell. 2007; 129:865-77.

29. Nellore A, Paziana K, Ma C, Tsygankova OM, Wang Y, Puttaswamy K, Iqbal AU, Franks SR, Lv Y, Troxel AB, Feldman MD, Meinkoth JL, Brose MS. Loss of Rap1GAP in papillary thyroid cancer. J Clin Endocrinol Metab. 2009; 94:1026-32.

30. Banerjee R, Mani RS, Russo N, Scanlon CS, Tsodikov A, Jing X, Cao Q, Palanisamy N, Metwally T, Inglehart RC, Tomlins S, Bradford C, Carey T, et al. The tumor suppressor gene rap1GAP is silenced by miR-101-mediated EZH2 overexpression in invasive squamous cell carcinoma. Oncogene. 2011; 30:4339-49.

31. Kim WJ, Gersey Z, Daaka Y. Rap1GAP regulates renal cell carcinoma invasion. Cancer Lett. 2012; 320:65-71.
32. Yang J, Mani SA, Weinberg RA. Exploring a new twist on tumor metastasis. Cancer Res. 2006; 66:4549-52.

33. Kim MC, Cui FJ, Kim Y. Hydrogen Peroxide Promotes Epithelial to Mesenchymal Transition and Stemness in Human Malignant Mesothelioma Cells. Asian Pac J Cancer Prev. 2013; 14:3625-30.

34. Wang HL, Zhou PY, Zhang Y, Liu P. Relationships Between Abnormal MMP2 Expression and Prognosis in Gastric Cancer: A Meta-Analysis of Cohort Studies. Cancer Biotherapy and Radiopharmaceuticals. 2014; 29:166-72.

35. Shen WS, Xi HQ, Wei B, Chen L. The prognostic role of matrix metalloproteinase 2 in gastric cancer: a systematic review with meta-analysis. Journal of Cancer Research and Clinical Oncology. 2014; 140:1003-9.

36. Shen W, Xi H, Wei B, Chen L. The prognostic role of matrix metalloproteinase 2 in gastric cancer: a systematic review with meta-analysis. J Cancer Res Clin Oncol. 2014; 140:1003-9. 